\title{
Analyzing a few Heuristic Algorithms Considering Machine Idle Time and Processing Time in Permutation Flow Shop Scheduling Problems
}

\author{
Baskar A \\ Research Scholar \\ SMBS, VIT University \\ Vellore, India
}

\author{
Anthony Xavior M \\ Professor \\ SMBS, VIT University \\ Vellore, India
}

\begin{abstract}
Flow Shop Scheduling has been an interesting field of research for over six decades. They are easy to formulate, yet difficult to solve. In a shop, there are ' $m$ ' machines arranged in series to process a set of ' $n$ ' jobs having different processing times. Each job has to pass through each machine, in order. The problem is to find a sequence of jobs to be processed in all the machines, so that a given performance parameter is optimized. The total number of schedules is $(n !)^{m}$. If the order of machines is not to be changed, the problem is simplified, and the overall number of solutions is reduced to $\mathrm{n}$ !. This problem is referred to a permutation flow shop scheduling problem, or PFSP in short. Starting from two machines, ' $n$ ' jobs, various Heuristics have been proposed over the years. After the invention of meta heuristics and evolutionary algorithms, and increased computational capabilities available today, finding optimal/ near optimal solutions become comparatively easier. In this paper, a few heuristic algorithms have been analyzed for makespan criterion considering machine idle time and processing time, by comparing the results with the well known CDS algorithm. Benchmark problems proposed by Taillard and Ruben Ruiz are used for the performance analysis.
\end{abstract}

\section{Keywords}

Heuristic Algorithm, Flow Shop Scheduling, Makespan, Benckmark Problems.

\section{INTRODUCTION}

A typical permutation flow shop scheduling problem involves the determination of the order of processing the required jobs with different processing times over different machines. The parameter to be optimized may be anything; the most popular one being, minimizing the makespan. The sequence is not changed and kept the same for all the machines. For the makespan minimization, the problem is NP-complete, if the number of machines is greater than two [1].

In the simplified PFSP, a number of assumptions are usually made [2]:

- All the jobs are independent, and available for processing at time zero.

- All the machines are continuously available.

- Each machine can process at most one job at a time, and each job can be processed only on one machine at a time.

- $\quad$ No preemption is allowed.
- Setup times are sequence independent, and are included in the processing times or are otherwise ignored.

- $\quad$ An infinite in-process storage buffer is assumed. If a given job needs an unavailable machine, then it joins a queue of unlimited size waiting for that machine.

Most of the optimization criteria are based on the completion times of the jobs at the different machines, which are denoted by $\mathrm{C}_{\mathrm{j}, \mathrm{i}}$, where $\mathrm{C}_{\mathrm{j}}$ refers the time at which job $\mathrm{j}$ is completed at the last machine. The completion times $\mathrm{C}_{\mathrm{j}, \mathrm{i}}$ can be easily calculated as follows:

For a given permutation $\pi$ of $n$ jobs, where $\pi_{(j)}$ denotes the job in the $\mathrm{j}$-th position, the completion times are calculated with the following expression:

$\mathrm{C} \pi_{(\mathrm{j}), \mathrm{i}}=\max \left[\mathrm{C} \pi_{(\mathrm{j}), \mathrm{i}-1}, \mathrm{C} \pi_{(\mathrm{j}-1), \mathrm{i}}\right]+\mathrm{p}_{\pi(\mathrm{j}), \mathrm{i}}$

This paper considers minimization of maximum completion time (makespan, $\mathrm{C}_{\max }$ ). Under this objective, the problem is denoted as $\mathrm{F} / \mathrm{prmu} / \mathrm{C}_{\max }$. Johnson's [3] paper mainly studied problems with two machines and provides the optimal solution. Slope Index algorithm was proposed by Palmer [4]. The CDS algorithm [5] proposed by Campbell et al. is based on the Johnson's algorithm and obtains the solution in $(\mathrm{m}-1)$ enumerations. The algorithm proposed by Dannenbring [6] reduces the ' $m$ ' machines, ' $n$ ' jobs problems in to a two machine, ' $n$ ' jobs problem and then uses the Johnson's rule to obtain the solution.

The NEH algorithm proposed by Nawaz et al. [7] is considered as the best among the simple heuristics, according to many studies conducted over the past decades, like by Turner and Booth [8], Taillard [9] and Ruiz and Maroto [10]. The number of enumerations in NEH algorithm is $[n(n+1) / 2]-$ 1 and applies insertion technique. The solution is built from first two jobs selected from the initial sequence obtained by arranging all the jobs in ascending order of their total processing times. The NEH Algorithm for the minimization of the makespan can be stated as follows:

Step 1:

Ordering the jobs by non increasing sums of processing times on the machines;

Step 2: 
Taking the first two jobs, and scheduling them in order to minimize the partial makespan, as if there were only these two jobs

Step 3:

For $\mathrm{k}=3$ to $\mathrm{n}$, Step 4 to be repeated

Step 4:

Inserting the $\mathrm{k}^{\text {th }}$ job at the place, which minimizes the partial makespan among the possible ones.

Total number of sequences to be enumerated $=[n(n+1) / 2]-1$.

Many researchers have analyzed and modified the NEH algorithm to improve the makespan. The authors have also proposed a family of NEH heuristics to improve the makespan further.

In addition to the processing time, machine idle time is also another important element in deciding the quality of the solution. Framinan et al. [11] considered 176 approaches for every objective function. Additionally, for every objective function, the RANDOM choice of a sequence is considered. For the 177 different approaches to generate the initial sequences, and for every combination of $\mathrm{n}=5,6,7$, $8,10,15,20,25,30,50,75,100$ jobs, and $\mathrm{m}=5,10,15,20$, 25 machines, 100 problem instances were generated. Therefore, 6000 problem instances were considered. The processing times were drawn randomly as integers from a discrete uniform distribution between 1 and 99 . It was concluded that for the makespan, the best five heuristic algorithms consists of the NEH-insertion approach using the following five initial sequences:

SUM PIJ / DECR (i.e. original NEH) Rank 1.

\section{SS SRA/2SRN / DECR Rank 3.}

\section{SS SRS/RCN / DECR Rank 4.}

\section{SS SRA/RCN / DECR Rank 5.}

\section{RA C3 / INCR Rank 7.}

It may be noted that in the top four cases, the decreasing order proves to be better.

SUM PIJ/ DECR: total processing times of the jobs, decreasing order (This indicator value is exactly the one used in the original NEH approach.)

SS SRA/2SRN/ DECR: sum of the absolute residuals with negative residuals weighted double, no carryover, decreasing order

SS SRS/RCN/ DECR: sum of the squared residuals with negative residual carryover, decreasing order

SS SRA/RCN/ DECR: sum of the absolute residuals with negative residual carryover, decreasing order

RA C3/INCR: sum of possible waiting time of jobs and idle time of machines, increasing order.

The sorting sequences are unlimited, and important were covered by Framinan et al. The authors have considered twenty three starting sequences, combining the total processing time, and the total Machine Idle Time. In all the cases, the insertion technique was used. However, unlike the original NEH, which selects the first two jobs as the initial sequence, the first and the last jobs, the middle two jobs and the last two jobs were also considered for the analysis.

This paper analyzes a few heuristic algorithms built using various combinations of the machine idle time and the processing time. They are different from the above discussed algorithms.

\section{HEURISTIC ALGORITHMS ANALYZED}

Many classic heuristics consider only the processing time, and give the result using different approaches. As all are aware of, the heuristic algorithms are approximate methods, and the PFSP is NP complete, it cannot be explicitly stated that a particular heuristic can always report an optimal/ near optimal solution. In addition to the NEH heuristic, which is the best among the available simple heuristics, CDS also performs reasonably better. The complexity level and the quality of the simple heuristics have been studied in detail by Taillard [9], and are briefly given below:

\begin{tabular}{|c|c|c|c|c|c|c|c|c|}
\hline \multicolumn{3}{|c|}{ Complexity } & \multicolumn{3}{|c|}{ Quality } & & & \\
\hline Problems & - & & 500 & 100 & 100 & 100 & 50 & 50 \\
\hline Jobs & $\mathrm{n}$ & & 9 & 10 & 20 & 20 & 40 & 50 \\
\hline Machines & $\mathrm{m}$ & & 10 & 10 & 10 & 20 & 10 & 10 \\
\hline Gupta & $\begin{array}{l}n \log (n) \\
n m\end{array}$ & + & 13.4 & 12.8 & 19.6 & 18.8 & 18.9 & 17.1 \\
\hline Johnson & $\begin{array}{l}n \log (n) \\
n m\end{array}$ & + & 10.9 & 11.8 & 16.7 & 16.8 & 17.3 & 16.3 \\
\hline RA & $\begin{array}{l}n \log (n) \\
n m\end{array}$ & + & 8.5 & 9.1 & 12.5 & 13.4 & 13.5 & 11.2 \\
\hline Palmer & $n \log (\mathrm{n})$ & + & 8.3 & 9.0 & 13.3 & 12.5 & 10.9 & 10.7 \\
\hline CDS & $\begin{array}{l}\mathrm{nm}^{2}+ \\
\mathrm{nlog}(\mathrm{n})\end{array}$ & & 4.5 & 5.2 & 9.7 & 8.6 & 9.9 & 9.3 \\
\hline $\mathrm{NEH}$ & $\mathrm{n}^{2} \mathrm{~m}$ & & 2.1 & 2.2 & 3.9 & 3.8 & 2.6 & 2.1 \\
\hline
\end{tabular}

NEH appears to be the best polynomial heuristic in practice. The CDS algorithm which is in the second place also performs better. The heuristics, RA or Palmer may also be useful when short computation times are required.

For the algorithms considered, the overall performance is assessed using a set of benchmark problems totaling 120 in number proposed by Taillard [12], and 250 in number proposed by Ruben Ruiz (2009). The processing time varies from 1 to 99 units and generated using a random number generator for a given seed. Codes were generated, run in an i5 PC with 4 GB RAM, and the following nine cases were analyzed:

1. CDS original algorithm

2. Total idle time in ascending order for all the machines, when a particular job is processed

3. Total idle time in descending order for all the machines when a particular job is processed

4. Total processing time in ascending order for a particular job

5. Total processing time in descending order for a particular job (NEH initial sequence)

6. Total Machine idle time + Total processing time $(\mathrm{IT}+\mathrm{PT})$, in ascending order

7. Total Machine idle time + Total processing time, in descending order 
8. Ratio of machine idle time to total processing time (IT/PT), in ascending order

9. Ratio of machine idle time to total processing time, in descending order

The jobs were scheduled based on the above and the corresponding makespans were computed. The method of computing a special category of total machine idle time and the usual total processing time is illustrated using an example given in Table 1.

Table 1, Example PFSP (5 machines, 5 jobs)

\begin{tabular}{lllrrl}
\hline & \multicolumn{5}{c}{ Jobs } \\
M/C' ' $m$ & \multicolumn{5}{c}{ Processing Times } \\
\hline 1 & 8 & 10 & 4 & 9 & 5 \\
2 & 5 & 4 & 6 & 3 & 7 \\
3 & 2 & 5 & 3 & 6 & 3 \\
4 & 8 & 4 & 2 & 5 & 8 \\
5 & 9 & 8 & 7 & 8 & 11 \\
\hline
\end{tabular}

Processing of Job 1: 0-8 units (M1) 8-13 units (M2) 13-15 units (M3) 15-23 units (M4) 23-32 units (M5).

Total machine Idle Time if job 1 is processed first: M1-0; M28; M3-13; M4-15; M5-23 and

Total Machine Idle Time: $(0+8+13+15+23)=59$ units. Similarly,

Total Machine idle time when Job No. 2 is processed first: 66 units

Total Machine idle time when Job No. 3 is processed first: 42 units

Total Machine idle time when Job No. 4 is processed first: 62 units

Total Machine idle time when Job No. 5 is processed first: 55 units

Total processing time for finishing Job no. $1:(8+5+2+8+9)=$ 32 units and

Total processing time for finishing Job no. 2: 31 units

Total processing time for finishing Job no. 3: 22 units

Total processing time for finishing Job no. 4: 31 units

Total processing time for finishing Job no. 5: 34 units

The total machine idle time array for the problem is, [59 66

426255 ] for the jobs from 1 to 5 respectively.

Similarly, the total processing time array is $\left[\begin{array}{llll}32 & 31 & 22 & 31\end{array}\right.$ 34].

In the algorithm using the ratio of machine idle time to total processing time (IT/PT), in ascending order (serial number 8 above), the ratio array is $\left[\begin{array}{lll}1.8438 & 2.1290 & 1.9091\end{array}\right.$ $2.00001 .6176]$. The ratio array is sorted in ascending order, and a sequence of $\left(\begin{array}{lllll}5 & 1 & 3 & 4 & 2\end{array}\right)$ with a makespan of 66 units (Table 2) is obtained. In the similar way, other procedures can easily be converted in to algorithms for computing the sequence and the corresponding makespan. The problem instances proposed by Taillard and Ruben Ruiz are used for the complete analysis of all the nine heuristic algorithms.
Table 2, Makespan for the sequence 5-1-3-4-2

\begin{tabular}{llllll}
\hline \multicolumn{5}{c}{ Machines } \\
Job & \multicolumn{5}{c}{ Processing Times } \\
\hline 5 & $0-5$ & $5-12$ & $12-15$ & $15-23$ & $23-34$ \\
1 & $5-13$ & $13-18$ & $18-20$ & $23-31$ & $34-43$ \\
3 & $13-17$ & $18-24$ & $24-27$ & $31-33$ & $.43-50$ \\
4 & $17-26$ & $26-29$ & $29-35$ & $35-40$ & $50-58$ \\
2 & $26-36$ & $36-40$ & $40-45$ & $45-49$ & $58-66$ \\
\hline
\end{tabular}

\section{ANALYSIS OF THE PERFORMANCE USING THE TAILLARD BENCHMARK PROBLEMS}

Initially, all the algorithms were tested for the makespan requirement. The complete results are shown for twenty Taillard problem instances in Tables 3 and 4. MS1 represents the makespan obtained using the first algorithm, the CDS algorithm and so on. It was found that, the CDS, and the algorithm based on the ratio of machine idle time to total processing time in ascending order, perform better in most of the cases for the makespan objective. Hence, other results are not listed. Only the results from these two algorithms are completely tabulated from Tables 5 to 8 , and the summary for the problems are shown in Table 11. It may be noted that Taillard listed the lower bounds for the 120 number problem instances and the lower bounds for the Ruben Ruiz problems are calculated by the authors. However, since the makespan values obtained from the CDS algorithm are taken as the reference values, these lower bounds are not considered anywhere in this paper.

\section{ANALYSIS OF THE PERFORMANCE USING THE TAILLARD BENCHMARK PROBLEMS}

Ruben Ruiz (2009) bench mark problems are available on web page for the research group "Sistemas de Optimización Aplicada SOA" [13] or Applied Optimization Systems: http://soa.iti.es. Taillard benchmark is composed of 12 groups of 10 instances each, totaling 120 instances. The groups, presented in the form of (jobs $x$ machines), are $\{20,50$, $100\} \times\{5,10,20\}, 200 \times\{10,20\}$ and $500 \times 20$. As this is incomplete as on today, in the sense that some combinations of ' $n$ ' and ' $m$ ' are missing, Ruben Ruiz problems are also used in addition. They were developed originally for no-idle flow shop scheduling problem, and are adapted for our permutation flow shop scheduling analysis. The benchmark has 250 instances, where 10 combinations of, $n=\{50,100$, $150,200,250,300,350,400,450,500\}$ jobs, and five combinations of, $\mathrm{m}=\{10,20,30,40,50\}$ machines. There are five replicates per combination. The processing times are uniformly distributed in the range [1,99], similar to the Taillard instances.

For this analysis, the results are tabulated in the Tables 9 and 10, and, the summary of the analysis is listed in Table 12, for all the 250 problem instances. 
Table 3, Makespans for first set of 10 problems

\begin{tabular}{llllllllll}
\hline Instance & MS1 & MS2 & MS3 & MS4 & MS5 & MS6 & MS7 & MS8 & MS9 \\
\hline ta001 & 1390 & 1377 & 1589 & 1472 & 1556 & 1377 & 1601 & 1384 & 1691 \\
ta002 & 1424 & 1469 & 1661 & 1538 & 1594 & 1481 & 1690 & 1437 & 1699 \\
ta003 & 1249 & 1463 & 1464 & 1499 & 1408 & 1473 & 1461 & 1191 & 1747 \\
ta004 & 1418 & 1466 & 1781 & 1640 & 1723 & 1547 & 1773 & 1420 & 1854 \\
ta005 & 1323 & 1395 & 1635 & 1437 & 1592 & 1387 & 1594 & 1338 & 1718 \\
ta006 & 1312 & 1372 & 1655 & 1457 & 1604 & 1373 & 1625 & 1313 & 1668 \\
ta007 & 1393 & 1516 & 1560 & 1504 & 1479 & 1547 & 1554 & 1360 & 1549 \\
ta008 & 1341 & 1426 & 1647 & 1422 & 1531 & 1415 & 1593 & 1300 & 1837 \\
ta009 & 1360 & 1352 & 1682 & 1585 & 1538 & 1396 & 1669 & 1426 & 1873 \\
ta010 & 1164 & 1325 & 1604 & 1365 & 1387 & 1329 & 1551 & 1208 & 1690 \\
\hline
\end{tabular}

Table 4, Makespans for last set of 10 problems

\begin{tabular}{llllllllll}
\hline Instance & MS1 & MS2 & MS3 & MS4 & MS5 & MS6 & MS7 & MS8 & MS9 \\
\hline ta111 & 28246 & 29075 & 31898 & 30694 & 30363 & 28914 & 31836 & 28149 & 33365 \\
ta112 & 29131 & 29928 & 31994 & 31435 & 31061 & 29898 & 31986 & 29326 & 34607 \\
ta113 & 28618 & 29700 & 31862 & 30786 & 30205 & 29826 & 32004 & 28187 & 34177 \\
ta114 & 29029 & 29119 & 32346 & 30546 & 31071 & 29263 & 32621 & 27884 & 33438 \\
ta115 & 28234 & 29139 & 32128 & 30570 & 30376 & 28963 & 32027 & 27891 & 34389 \\
ta116 & 28687 & 28885 & 32264 & 30072 & 30811 & 28735 & 32141 & 28257 & 33811 \\
ta117 & 28322 & 28624 & 32191 & 30640 & 30698 & 28834 & 32251 & 28029 & 33796 \\
ta118 & 28897 & 29223 & 32640 & 30804 & 30658 & 29065 & 32296 & 28632 & 33635 \\
ta119 & 28294 & 28639 & 32226 & 30239 & 30442 & 28718 & 32228 & 27775 & 33784 \\
ta120 & 28710 & 28966 & 31630 & 30070 & 30936 & 29053 & 31827 & 28122 & 33874 \\
\hline
\end{tabular}

Table 5, Twenty Jobs PFSPs

\begin{tabular}{|c|c|c|c|c|c|c|c|c|}
\hline Instance & CDS & IT/PT & Instance & CDS & IT/PT & Instance & CDS & IT/PT \\
\hline ta001 & 1390 & 1384 & $\operatorname{ta} 011$ & 1757 & 1758 & ta021 & 2559 & 2777 \\
\hline ta002 & 1424 & 1437 & $\mathrm{ta} 012$ & 1854 & 1875 & $\operatorname{ta} 022$ & 2285 & 2335 \\
\hline ta003 & 1249 & 1191 & $\mathrm{ta} 013$ & 1645 & 1699 & $\mathrm{ta} 023$ & 2565 & 2678 \\
\hline ta004 & 1418 & 1420 & $\mathrm{ta} 014$ & 1547 & 1584 & $\mathrm{ta} 024$ & 2434 & 2496 \\
\hline ta005 & 1323 & 1338 & $\mathrm{ta} 015$ & 1558 & 1629 & $\mathrm{ta} 025$ & 2506 & 2652 \\
\hline ta006 & 1312 & 1313 & $\operatorname{ta} 016$ & 1591 & 1500 & ta026 & 2422 & 2630 \\
\hline ta007 & 1393 & 1360 & $\operatorname{ta} 017$ & 1630 & 1637 & $\operatorname{ta} 027$ & 2489 & 2519 \\
\hline ta008 & 1341 & 1300 & $\operatorname{ta} 018$ & 1788 & 1846 & $\operatorname{ta} 028$ & 2362 & 2421 \\
\hline ta009 & 1360 & 1426 & $\operatorname{ta} 019$ & 1720 & 1826 & $\operatorname{ta} 029$ & 2414 & 2704 \\
\hline ta010 & 1164 & 1208 & $\mathrm{ta} 020$ & 1884 & 1882 & $\operatorname{ta} 030$ & 2469 & 2590 \\
\hline
\end{tabular}


Table 6, Fifty Jobs PFSPs

\begin{tabular}{lll|lll|lll}
\hline Instance & CDS & IT/PT & Instance & CDS & IT/PT & Instance & CDS & IT/PT \\
\hline ta031 & 2816 & 2743 & ta041 & 3421 & 3415 & ta051 & 4328 & 4291 \\
ta032 & 3032 & 2943 & ta042 & 3246 & 3286 & ta052 & 4216 & 4250 \\
ta033 & 2703 & 2698 & ta043 & 3280 & 3403 & ta053 & 4189 & 4214 \\
ta034 & 2884 & 2913 & ta044 & 3393 & 3461 & ta054 & 4280 & 4278 \\
ta035 & 3038 & 2945 & ta045 & 3375 & 3366 & ta055 & 4122 & 4382 \\
ta036 & 3031 & 3065 & ta046 & 3400 & 3404 & ta056 & 4267 & 4202 \\
ta037 & 2969 & 2821 & ta047 & 3520 & 3543 & ta057 & 4134 & 4355 \\
ta038 & 2835 & 2779 & ta048 & 3387 & 3275 & ta058 & 4262 & 4394 \\
ta039 & 2784 & 2706 & ta049 & 3251 & 3278 & ta059 & 4212 & 4390 \\
ta040 & 2942 & 2915 & ta050 & 3429 & 3341 & ta060 & 4270 & 4239 \\
\hline
\end{tabular}

Table 7, Hundred Jobs PFSPs

\begin{tabular}{|c|c|c|c|c|c|c|c|c|}
\hline Instance & CDS & IT/PT & Instance & CDS & IT/PT & Instance & CDS & IT/PT \\
\hline ta061 & 5592 & 5716 & ta071 & 6209 & 6028 & ta081 & 6920 & 7158 \\
\hline ta062 & 5563 & 5328 & ta072 & 5873 & 5877 & ta082 & 6977 & 7173 \\
\hline ta063 & 5493 & 5299 & $\operatorname{ta} 073$ & 6024 & 6014 & ta083 & 7229 & 7105 \\
\hline ta064 & 5273 & 5074 & $\mathrm{ta} 074$ & 6377 & 6416 & $\mathrm{ta} 084$ & 7062 & 7117 \\
\hline ta065 & 5461 & 5438 & ta075 & 6018 & 6000 & ta085 & 7113 & 7304 \\
\hline ta066 & 5259 & 5241 & ta076 & 5744 & 6091 & ta086 & 7283 & 7019 \\
\hline ta067 & 5557 & 5275 & ta077 & 6201 & 6280 & $\mathrm{ta} 087$ & 7147 & 7554 \\
\hline ta068 & 5387 & 5221 & ta078 & 6234 & 6042 & ta088 & 7235 & 7646 \\
\hline ta069 & 5758 & 5571 & ta079 & 6349 & 6139 & ta089 & 7196 & 7282 \\
\hline ta070 & 5723 & 5363 & $\operatorname{ta} 080$ & 6387 & 6365 & $\operatorname{ta} 090$ & 7164 & 7279 \\
\hline
\end{tabular}

Table 8, Two Hundred and Five Hundred Jobs PFSPs

\begin{tabular}{lll|lll|lll}
\hline Instance & CDS & IT/PT & Instance & CDS & IT/PT & Instance & CDS & IT/PT \\
\hline ta091 & 11609 & 11476 & ta101 & 12432 & 12835 & ta111 & 28246 & 28149 \\
ta092 & 11357 & 11059 & ta102 & 12542 & 12940 & ta112 & 29131 & 29326 \\
ta093 & 11669 & 11287 & ta103 & 12783 & 12813 & ta113 & 28618 & 28187 \\
ta094 & 11379 & 11217 & ta104 & 12631 & 12715 & ta114 & 29029 & 27884 \\
ta095 & 11352 & 11062 & ta105 & 12538 & 12562 & ta115 & 28234 & 27891 \\
ta096 & 11318 & 10870 & ta106 & 12498 & 12352 & ta116 & 28687 & 28257 \\
ta097 & 11608 & 11363 & ta107 & 12711 & 12760 & ta117 & 28322 & 28029 \\
ta098 & 11501 & 11259 & ta108 & 12610 & 12851 & ta118 & 28897 & 28632
\end{tabular}


International Journal of Applied Information Systems (IJAIS) - ISSN : 2249-0868

Foundation of Computer Science FCS, New York, USA

Volume 6-No. 4, September 2013 - www.ijais.org

\begin{tabular}{ccc|ccc|ccc} 
ta099 & 11252 & 10923 & ta109 & 12639 & 12624 & ta119 & 28294 & 27775 \\
ta100 & 11509 & 11102 & ta110 & 12949 & 12751 & ta120 & 28710 & 28122 \\
\hline
\end{tabular}

Table 9, 1 - 150 number of Ruben Ruiz Problems

\begin{tabular}{|c|c|c|c|c|c|c|c|c|}
\hline Instance & CDS & IT/PT & Instance & CDS & IT/PT & Instance & CDS & IT/PT \\
\hline \multirow[t]{25}{*}{50 jobs } & 3409 & 3405 & \multirow[t]{25}{*}{150 jobs } & 8862 & 8742 & \multirow[t]{25}{*}{250 jobs } & 14621 & 14237 \\
\hline & 3463 & 3445 & & 8628 & 8363 & & 14039 & 13583 \\
\hline & 3412 & 3424 & & 8646 & 8414 & & 13802 & 13735 \\
\hline & 3381 & 3357 & & 8675 & 8641 & & 14118 & 13764 \\
\hline & 3672 & 3712 & & 8576 & 8432 & & 14246 & 13676 \\
\hline & 4377 & 4428 & & 9933 & 10009 & & 15307 & 15439 \\
\hline & 4295 & 4481 & & 9851 & 10367 & & 15101 & 15501 \\
\hline & 4280 & 4440 & & 9895 & 10192 & & 15278 & 15349 \\
\hline & 4252 & 4334 & & 9634 & 9977 & & 15353 & 15290 \\
\hline & 4319 & 4320 & & 9939 & 10067 & & 15360 & 15498 \\
\hline & 5249 & 5554 & & 11203 & 11351 & & 16752 & 16405 \\
\hline & 5136 & 5424 & & 10979 & 11126 & & 16510 & 16905 \\
\hline & 4930 & 5274 & & 11070 & 11334 & & 16433 & 16599 \\
\hline & 5061 & 5126 & & 11287 & 11420 & & 16594 & 16821 \\
\hline & 5109 & 5206 & & 10998 & 11196 & & 16594 & 16816 \\
\hline & 5718 & 5998 & & 11998 & 12333 & & 17486 & 17641 \\
\hline & 5709 & 5979 & & 11845 & 11985 & & 17684 & 18075 \\
\hline & 5908 & 6096 & & 11734 & 11881 & & 17888 & 17834 \\
\hline & 5845 & 5983 & & 11788 & 12175 & & 17825 & 17987 \\
\hline & 5779 & 5934 & & 12179 & 12291 & & 17831 & 17802 \\
\hline & 6494 & 6636 & & 12939 & 13402 & & 18936 & 18860 \\
\hline & 6695 & 6884 & & 12835 & 13149 & & 18855 & 18826 \\
\hline & 6466 & 6659 & & 12761 & 13272 & & 18552 & 19051 \\
\hline & 6565 & 6707 & & 12987 & 13112 & & 18603 & 19092 \\
\hline & 6646 & 6839 & & 12937 & 13203 & & 18737 & 19086 \\
\hline \multirow[t]{22}{*}{100 jobs } & 6047 & 5936 & \multirow[t]{22}{*}{200 jobs } & 11252 & 11185 & \multirow[t]{22}{*}{300 jobs } & 16496 & 16113 \\
\hline & 6161 & 6035 & & 11602 & 11378 & & 16727 & 16330 \\
\hline & 5923 & 6111 & & 11502 & 11258 & & 16964 & 16730 \\
\hline & 5877 & 5870 & & 11633 & 11508 & & 16809 & 16281 \\
\hline & 6233 & 6251 & & 11296 & 10996 & & 16771 & 16626 \\
\hline & 7189 & 7308 & & 12724 & 12560 & & 18141 & 17708 \\
\hline & 7189 & 7115 & & 12727 & 12573 & & 18010 & 18477 \\
\hline & 7143 & 7229 & & 12830 & 12667 & & 18043 & 17877 \\
\hline & 7017 & 7080 & & 12704 & 12665 & & 17724 & 17894 \\
\hline & 7432 & 7632 & & 12554 & 12999 & & 18459 & 18144 \\
\hline & 8112 & 8225 & & 13584 & 13898 & & 19581 & 19419 \\
\hline & 8187 & 8008 & & 13733 & 13793 & & 19241 & 19339 \\
\hline & 8395 & 8511 & & 13738 & 13811 & & 19624 & 19642 \\
\hline & 8270 & 8555 & & 13986 & 14017 & & 19468 & 19909 \\
\hline & 8419 & 8572 & & 13748 & 13657 & & 19435 & 19660 \\
\hline & 8884 & 9203 & & 14873 & 15167 & & 20407 & 20707 \\
\hline & 8914 & 9135 & & 14936 & 15567 & & 20693 & 20988 \\
\hline & 8894 & 9183 & & 14785 & 14935 & & 20651 & 21365 \\
\hline & 8999 & 9280 & & 14638 & 15024 & & 20480 & 21197 \\
\hline & 8926 & 9073 & & 14866 & 14913 & & 20674 & 20646 \\
\hline & 9876 & 10253 & & 15780 & 16336 & & 21968 & 22049 \\
\hline & 9844 & 9974 & & 15872 & 16239 & & 21520 & 21981 \\
\hline
\end{tabular}


International Journal of Applied Information Systems (IJAIS) - ISSN : 2249-0868

Foundation of Computer Science FCS, New York, USA

Volume 6-No. 4, September 2013 - www.ijais.org

\begin{tabular}{ll|ll|ll}
9775 & 9753 & 15841 & 15841 & 21819 & 21965 \\
9642 & 10189 & 15785 & 16023 & 21743 & 22048 \\
9747 & 10079 & 15713 & 16190 & 21829 & 21487 \\
\hline
\end{tabular}

Table 10, 151 - 250 number of Ruben Ruiz Problems

\begin{tabular}{|c|c|c|c|c|c|c|c|c|}
\hline Instance & CDS & IT/PT & Instance & CDS & IT/PT & Instance & CDS & IT/PT \\
\hline \multirow[t]{25}{*}{350 jobs } & 19991 & 19443 & \multirow[t]{25}{*}{400 jobs } & 22550 & 22357 & \multirow[t]{25}{*}{450 jobs } & 24109 & 23622 \\
\hline & 19173 & 18661 & & 21634 & 21156 & & 23983 & 23306 \\
\hline & 19314 & 18880 & & 22080 & 21831 & & 24084 & 23670 \\
\hline & 19179 & 18972 & & 21733 & 21331 & & 24640 & 24134 \\
\hline & 19245 & 19166 & & 22280 & 21367 & & 24658 & 23983 \\
\hline & 20713 & 20425 & & 23523 & 23233 & & 26345 & 25859 \\
\hline & 21006 & 20495 & & 23756 & 24030 & & 25752 & 25330 \\
\hline & 20856 & 20720 & & 23411 & 23426 & & 26124 & 26179 \\
\hline & 20861 & 20757 & & 23590 & 23209 & & 26068 & 25375 \\
\hline & 20492 & 20324 & & 23562 & 23543 & & 26233 & 25906 \\
\hline & 21930 & 21810 & & 24993 & 25002 & & 27729 & 27151 \\
\hline & 22010 & 22016 & & 24891 & 24816 & & 27338 & 27302 \\
\hline & 22084 & 23045 & & 24806 & 24759 & & 27577 & 27272 \\
\hline & 22212 & 22096 & & 24933 & 24583 & & 27106 & 27216 \\
\hline & 22123 & 22310 & & 24988 & 25353 & & 27659 & 27413 \\
\hline & 23590 & 23600 & & 26262 & 26570 & & 28864 & 28823 \\
\hline & 23183 & 23015 & & 25960 & 25998 & & 29096 & 28881 \\
\hline & 23184 & 22950 & & 26154 & 26322 & & 28894 & 28752 \\
\hline & 23080 & 23235 & & 25945 & 26301 & & 28939 & 28902 \\
\hline & 23175 & 22934 & & 26029 & 26141 & & 29013 & 29122 \\
\hline & 24217 & 24580 & & 27566 & 28006 & & 30415 & 30709 \\
\hline & 24317 & 24882 & & 27226 & 27764 & & 30345 & 30440 \\
\hline & 24514 & 24735 & & 27410 & 27556 & & 30086 & 29834 \\
\hline & 24361 & 24629 & & 27258 & 28047 & & 30219 & 30336 \\
\hline & 24373 & 24525 & & 27301 & 27380 & & 30317 & 30659 \\
\hline 500 jobs & 27231 & 27314 & 500 jobs & 29144 & 28721 & 500 jobs & 29999 & 30285 \\
\hline \multirow[t]{4}{*}{$10 \mathrm{~m} / \mathrm{c}$} & 27636 & 27842 & \multirow[t]{4}{*}{$20 \mathrm{~m} / \mathrm{c}$} & 29039 & 28709 & \multirow[t]{4}{*}{$30 \mathrm{~m} / \mathrm{c}$} & 30382 & 30314 \\
\hline & 27399 & 26845 & & 28842 & 28143 & & 30425 & 30213 \\
\hline & 27118 & 26247 & & 28638 & 28645 & & 29884 & 29692 \\
\hline & 26519 & 26145 & & 29210 & 27977 & & 30379 & 30010 \\
\hline 500 jobs & 31549 & 31730 & 500 jobs & 32868 & 33043 & & & \\
\hline \multirow[t]{4}{*}{$40 \mathrm{~m} / \mathrm{c}$} & 31820 & 31824 & \multirow[t]{4}{*}{$50 \mathrm{~m} / \mathrm{c}$} & 33078 & 32742 & & & \\
\hline & 31325 & 31461 & & 33080 & 33036 & & & \\
\hline & 31499 & 31200 & & 32836 & 33246 & & & \\
\hline & 31795 & 31779 & & 32943 & 33113 & & & \\
\hline
\end{tabular}


Table 11, Summary for the Taillard Problems

\begin{tabular}{|c|c|c|c|c|c|c|c|}
\hline \multicolumn{8}{|c|}{ Jobs } \\
\hline & & $\mathrm{M} / \mathrm{Cs}$ & 20 & 50 & 100 & 200 & 500 \\
\hline CDS & 9 & \multirow[t]{2}{*}{5} & 6 & 2 & 1 & - & - \\
\hline IT/PT & 21 & & 4 & 8 & 9 & - & - \\
\hline $\mathrm{CDS}$ & 18 & \multirow[t]{2}{*}{10} & 8 & 6 & 4 & 0 & - \\
\hline IT/PT & 22 & & 2 & 4 & 6 & 10 & - \\
\hline CDS & 32 & \multirow[t]{2}{*}{20} & 10 & 6 & 8 & 7 & 1 \\
\hline \multirow[t]{3}{*}{ IT/PT } & 18 & & 0 & 4 & 2 & 3 & 9 \\
\hline & & CDS & 24 & 14 & 13 & 7 & 1 \\
\hline & & IT/PT & 6 & 16 & 17 & 13 & 9 \\
\hline
\end{tabular}

Table 12, Summary for the Ruben Ruiz Problems

\begin{tabular}{|c|c|c|c|c|c|c|c|c|c|c|c|c|}
\hline \multicolumn{13}{|c|}{ Jobs } \\
\hline & & $\mathrm{M} / \mathrm{Cs}$ & 50 & 100 & 150 & 200 & 250 & 300 & 350 & 400 & 450 & 500 \\
\hline CDS & 6 & \multirow[t]{2}{*}{10} & 2 & 2 & 0 & 0 & 0 & 0 & 0 & 0 & 0 & 2 \\
\hline IT/PT & 44 & & 3 & 3 & 5 & 5 & 5 & 5 & 5 & 5 & 5 & 3 \\
\hline CDS & 25 & \multirow[t]{2}{*}{20} & 5 & 4 & 5 & 1 & 4 & 2 & 0 & 2 & 1 & 1 \\
\hline $\mathrm{IT} / \mathrm{PT}$ & 25 & & 0 & 1 & 0 & 4 & 1 & 3 & 5 & 3 & 4 & 4 \\
\hline $\mathrm{CDS}$ & 33 & \multirow[t]{2}{*}{30} & 5 & 4 & 5 & 4 & 4 & 4 & 3 & 2 & 1 & 1 \\
\hline $\mathrm{IT} / \mathrm{PT}$ & 17 & & 0 & 1 & 0 & 1 & 1 & 1 & 2 & 3 & 4 & 4 \\
\hline CDS & 38 & \multirow[t]{2}{*}{40} & 5 & 5 & 5 & 5 & 3 & 4 & 2 & 5 & 1 & 3 \\
\hline $\mathrm{IT} / \mathrm{PT}$ & 12 & & 0 & 0 & 0 & 0 & 2 & 1 & 3 & 0 & 4 & 2 \\
\hline CDS & 43 & \multirow[t]{2}{*}{50} & 5 & 4 & 5 & 5 & 3 & 4 & 5 & 5 & 4 & 3 \\
\hline \multirow[t]{3}{*}{ IT/PT } & 8 & & 0 & 1 & 0 & 1 & 2 & 1 & 0 & 0 & 1 & 2 \\
\hline & & CDS & 22 & 19 & 20 & 15 & 14 & 14 & 10 & 14 & 7 & 10 \\
\hline & & IT/PT & 3 & 6 & 5 & 11 & 11 & 11 & 15 & 11 & 18 & 15 \\
\hline
\end{tabular}

\section{CONCLUSION}

Table 3 to Table 8 show the results obtained for the nine heuristic algorithms when Taillard bench mark problems are used. To compute the sequence and the corresponding makespan, these algorithms use different combinations of total machine idle time while processing a job and total processing time of the corresponding job. The codes were run in an i5 PC with 4GB RAM.

From Table 3 and 4, it is clear that except the CDS and the (Idle Time/ Processing Time) based algorithm, all others fail to produce optimal/ near optimal results. Hence, the other values are ignored in other Tables.

It may be noted that for the 20x20 PFSPs, CDS completely outperforms the other one for all ten problem instances. Whereas, for the 10x200 problems set, the other algorithm gives better makespans than the CDS for all the ten instances. CDS performs better in the problem sets $10 \times 20,20 \times 50$, $20 \times 100$ and 20x200; and at the same time, problem sets 5x50, $5 \times 100$ and $20 \times 500$ are dominated by the other one. The performance is more or less same for other problem instances. In total, CDS produces better makespans in 59 instances, and the IT/PT heuristic algorithm, in 61 instances.

The case is different in case of Ruben Ruiz problems. Out of the 250 problems, CDS reports better makespans for 145 problems, whereas, IT/PT accounts for 106 only. Unlike the Taillard problems where, the lower bounds are listed along with the problems, they are not given for the Ruben Ruiz problems. The authors have computed the lower bounds. It can be seen that, ten machine problems are dominated by the IT/PT algorithm whereas; forty and fifty machine problems are dominated by the CDS algorithm.

If the summary in both the cases are analyzed, following conclusions could be drawn:

For Taillard Problems:

- $\quad$ IT/PT algorithm fares better in the case of $n \times\{5$, $10\}$ group of problems. Also, it outperforms CDS in $\{50,100,200,500\} \times \mathrm{m}$ problems.

- The CDS algorithm reports better makespans in the other cases.

For Ruben Ruiz Problems:

- The performance of IT/PT is better for $\mathrm{n} \times\{10,20\}$ group of problems. Also, it reports better makespans for $\{350,400,450,500\} \times \mathrm{m}$ problems.

- CDS algorithm reports better results in all the other sets of problems.

In general, for all the permutation flow shop problems with less number of machines and more number of jobs, the IT/PT algorithm can be effectively used. It may be noted that, the CDS algorithm selects the makespan from (m-1) enumerations. However, the IT/PT algorithm computes the makespan in a single enumeration and hence the computing time is less when compared to the CDS algorithm. In spite of less number of enumerations, the performance is comparable with the CDS algorithm in quite a good number of problems. 
Hence, for smaller problems involving manual computations, the better choice is the algorithm based on the IT/PT ratio. For computations involving computers, both can be used for the makespan and the better sequence can be selected from the results. Also, the result could be used as the candidate solution for refining the solution further, using any meta heuristic.

\section{REFERENCES}

[1] Rinnooy Kan, A. H. G. 1976. Machine Scheduling Problems: Classification, Complexity, and Computations (The Hague: Martinus Nijhoff).

[2] Baker, K. R. 1974. Introduction to Sequencing and Scheduling. John Wiley \& Sons, New York.

[3] Johnson, S. M. 1954. "Optimal two- and three-stage production schedules with setup times included", Naval Research Logistics Quarterly, vol. 1, pp. 61-68.

[4] Palmer, D. S. 1965. "Sequencing jobs through a multistage process in the minimum total time - a quick method of obtaining a near optimum", Operational Research Quarterly, vol. 16, No.1, pp. 101-107.

[5] Camperll, H. G., Campbell, Dudek, R. A., and Smith, M. L. 1970. "A heuristic algorithm for the $n$ job, $m$ machine sequencing problem", Management Science, vol. 16, No. 10, pp. B630-B637.

[6] Dannenbring, D. G. 1977. "An evaluation of flow shop sequencing heuristics", Management Science, vol. 23, No. 11, pp. 1174-1182.
[7] Nawaz, M, Enscore, Jr, E. E., and Ham, I. (1983). “A heuristic algorithm for the m- machine, n-job flow-shop sequencing problem", OMEGA, The International Journal of Management Science, vol. 11, No. 1, pp. 9195.

[8] Turner, S. and Booth, D. 1977. "Comparison of heuristics for flow shop sequencing", OMEGA, The International Journal of Management Science, vol. 15, No. 1, pp. 75-78.

[9] Taillard, E. 1990. "Some efficient heuristic methods for the flow shop sequencing problem", European Journal of Operational Research, vol. 47, pp. 67-74.

[10] Ruiz, R. and Maroto, C. 2005. "A comprehensive review and evaluation of permutation flowshop heuristics", European Journal of Operational Research, vol. 165, pp. 479-494.

[11] Framinan, J. M., Leisten, R., and Rajendran, C. 2003. "Different initial sequences for the heuristic of Nawaz, Enscore and Ham to minimize makespan, idletime or flowtime in the static permutation flowshop sequencing problem", International Journal of Production Research, vol. 41, No. 1, pp. 121-148.

[12] Taillard, E. 1993. "Benchmarks for basic scheduling problems", European Journal of Operational Research, vol. 64 , pp. 278-285.

[13] Ruben Ruiz problems available at http://soa.iti.es 